\title{
Design and Implementation of Real Time RMS Measurement System based on Wavelet Transform Using adsPIC-type Microcontroller
}

\author{
Hossam Eddine Guia \\ Department of Mechanical Engineering, Faculty of Technology, El-Oued University, 39000 El-Oued, Algeria \\ Email: guia-hossameddine@univ-eloued.dz
}

\begin{abstract}
Ammar Soukkou
Renewable Energy Laboratory, Faculty of Sciences and Technology, Electronics Department, Jijel University, P.O. Box .98, Ouled-Aissa, Jijel, 18000, Algeria.

Email: soukkou.amr@gmail.com; soukkou.ammar@univ-jijel.dz

\section{Redha Meneceur}

Department of Mechanical Engineering, Faculty of Technology, El-Oued University, 39000 El-Oued, Algeria

Email: meneceurredha22@yahoo.fr
\end{abstract}

\author{
Abdelkrim Mohrem \\ M'Hamed Bougara University of Boumerdés, Boumerdés, Algeria. \\ Email: mohrem.abdelkrim@gmail.com
}

Received: 22 March 2020; Accepted: 20 August 2020; Published: 08 December 2020

\begin{abstract}
This paper presents a design and implementation of Root Mean Square (RMS) measurement system based on fast discrete Wavelet using a dsPIC-type microcontroller. For data acquisition, two sensors have been used such as the voltage divider for sensing voltage and the Hall Effect sensor for sensing the current. The proposed method has the realtime calculation advantages and can be used in sinusoidal and non-sinusoidal electrical power systems. The results of calculations have been verified using MATLAB and Proteus ISIS simulations. It has been proved that the Wavelet transform measuring technique is more accurate as it takes in consideration all the harmonics in the analyzed signal and provides temporal information, which is absent in other transforms or not directly available in the Fourier transform.
\end{abstract}

Index Terms: Wavelet transform, Root mean square, Electrical energy meter, Real-time measurement system, Digital Signal Processing, microcontroller.

\section{Introduction}

In the last years, the interest for power quality has increased both to utilities and their customers. One of the major power system problems is steady-state waveform distortion due to harmonics. Harmonics are produced by variable speed drives, arc furnaces, three phase rectifiers with diode, personal computers, and other nonlinear devices. Since, harmonics can severely degrade the performance of power supplies and their connected equipment; it is important and necessary to always monitor harmonic parameters such as voltage, current, and power.

Nowadays, power grid; non-linear loads, such as power supply based on power electronics components; arching furnaces in steel mills; computer equipment in call centers; or six pulse rectifiers in motor drives are common harmonic distortion sources in both of current and voltage waveform and are used wide spread. Since harmonics have effects on electrical quantities measurement, they must be carefully monitored. In this situation, using an effective and fast method is necessary to take in consideration those distortions when the current and voltage RMS values are being calculated. For this purpose, Fourier-based methods have been proposed [1]. Although these methods have shown a very good performance; they are not suitable for non-stationary waveforms analysis. Wavelet transforms (WT) based methods have been proposed to overcome the drawbacks of the Fourier-based methods. Despite the fact that the computational cost of the Wavelet transform is higher than the Fourier transform. The wavelet transform has received great importance in the last years on the power system analysis because the multi-resolution analysis presents proprieties good for the transient signal analysis. Continuous and discrete Wavelet transforms (CWTs and DWTs) are promising tools that have 
been used in non-stationary signals analysis, and several recent papers have proposed the use of Wavelets for power system parameters calculation [2-11]. For example, E. Y. Hamid et al. [6] proposed an approach based on wavelet packet transform (WPT) for RMS values of voltage and power measurements. The algorithm can simultaneously measure the distribution of the RMS of voltage or current and power with respect to individual frequency bands from the Wavelet coefficients associated with each voltage current pair. The advantage of the WPT is that it can decompose a power system waveform into uniform frequency bands, which are important for identification of harmonic components and measurement of harmonic parameters. The algorithm is validated using simulated Waveforms. In [7], a novel approach based on Wavelet Packet Transform was presented to measure RMS value and phase angle of the fundamental harmonic. The analytical expressions were derived and using a Graphical User Interface (GUI) designed and experimental works the validity of analytical expressions was demonstrated. In [8] an RMS voltage estimator that eludes the inherent uncertainty of complex arithmetic operations related to the discretized RMS algorithm has been presented. The authors proposed an approximation to the problem by means of an RMS value estimator that uses as a base a microcontroller of basic range and low economic cost and algorithms of calculation of very low computational cost that elude complex arithmetic operation to controllers, such as powers or roots. The importance of selection of mother Wavelet for analyzing stationary disturbances using DWT has been presented in [9]. Signals with stationary disturbances of various frequencies are generated in these works using MATLAB. The analysis of these signals is done using various mother Wavelets like Daubechies and bi-orthogonal Wavelets and the measured RMS value of stationary disturbance is obtained. The measured value obtained by DWT is compared with the exact RMS value of the frequency component and the percentage differences are presented which helps to select optimum mother Wavelet. In [10], a new method has been proposed for fundamental power calculation based on a Wavelet transform with pre-processing by the Hilbert transform. The proposed method increases the frequencies of all harmonics by half of the main frequencies and locates both odd and even harmonics in the centres of the extracted frequency bands. In [11], the authors presented a review on main application of Wavelet transform in electric power systems. The study areas have been classified as power system protection, power quality disturbances, power system transient, partial discharge, load forecasting, faults detection, and power system measurement.

Taking into consideration the previous discussion, we notice that there has not been work on applying the Wavelet transform for real time measurement systems which is required in all energy meters. To solve this problem we propose in this paper an RMS calculation system based on fast discrete Wavelet and implemented by using a dsPIC-type microcontroller which has the advantage of real-time calculation of the distorted stationary and non-stationary signals. Firstly, the system is simulated with MATLAB to verify the validity of the proposed system. Next, the system is implemented in a dsPIC-type microcontroller using Proteus ISIS to validate the feasibility and effectiveness of the proposed approach. The MATLAB simulation results have been compared to the analytic results and have shown a good precision where the error does not exceed $2 \%$ in all simulated cases.

The remaining part of this paper is organized as follows. Section 2 considers the Wavelet transform clarification. Section 3 examines the RMS value definition in the Wavelet domain. The results of MATLAB and Proteus ISIS simulations are presented in Section 4 and 5. Finally, the conclusion is drawn in Section 5.

\section{Wavelet Transform}

A Wavelet is a "small wave", which has its energy concentrated in time to give a tool for the analysis of transient, non-stationary, or time-varying phenomena. We will take Wavelets and use them in a series expansion of signals or functions much the same way a Fourier series uses the wave or sinusoid to represent a signal or function [12].

In general, the Wavelet transform is concluded in two DWT and CWT forms. These forms are introduced in the next subsection.

\section{A. Continuous Wavelet Transform}

The CWT of a real signal $f(t)$ is computed as

$$
W_{f}=\int_{-\infty}^{+\infty} f(t) \frac{1}{\sqrt{|a|}} \psi *\left(\frac{t-b}{a}\right) d t
$$

where $a \in \square$ with $a \neq 0$ and $b \in \square$ represent the dilation and translation parameters, respectively. $\psi^{*}(t)$ is the complex conjugate of mother Wavelet $\psi(t)$ where mother Wavelet is a function with limited period and zero average [13]. 


\section{B. Discrete Wavelet transform}

The passage from the CWT to DWT is done by the discretization of the dilatation and translation parameters as follows [14]

$$
\left\{\begin{array}{l}
a=2^{j} \\
b=2^{j} k
\end{array} \quad k=1,2,3, \cdots \text { and } j=k=1,2,3, \cdots\right.
$$

By definition, the DWT of a real signal $f(t)$ is computed as follows

$$
D W_{f}=\int_{-\infty}^{+\infty} f(t) \frac{1}{\sqrt{2^{j}}} \psi *\left(\frac{t-\left(k 2^{j}\right)}{2^{j}}\right) d t
$$

The Wavelet basis functions can be implemented as a finite impulse response filter to get a multi-resolution analysis (MRA) [15]. It is noted that S. Mallat [16] has been introduced the MRA in the Wavelet theory for implementation of DWT and unified the structure of Wavelet orthogonal basis. The author, also, proved that the discrete signals could be decomposed and reconstructed according to the Wavelet transform.

The Wavelet decomposition process is depicted in Fig. 1. The original signal $f(n)$ consists of $2^{N}$ sampling points which is the input signal. Firstly, the operation of convolution is performed to the input signal $f(n)$ respectively with high-pass $g(n)$ and low-pass $h(n)$ filters, and then the results are down sampled by a factor 2 . The output of the highpass filter represents the Detail coefficients $D_{l}$ and contains the high frequencies components of the original signal. On the other hand the low-pass filter output represents the Approximation coefficients $A_{l}$ and contains the low frequencies components of the original signal. From the second level the Approximation and Detail coefficients are obtained from the decomposition of the approximation of the previous level and so on until the last level.

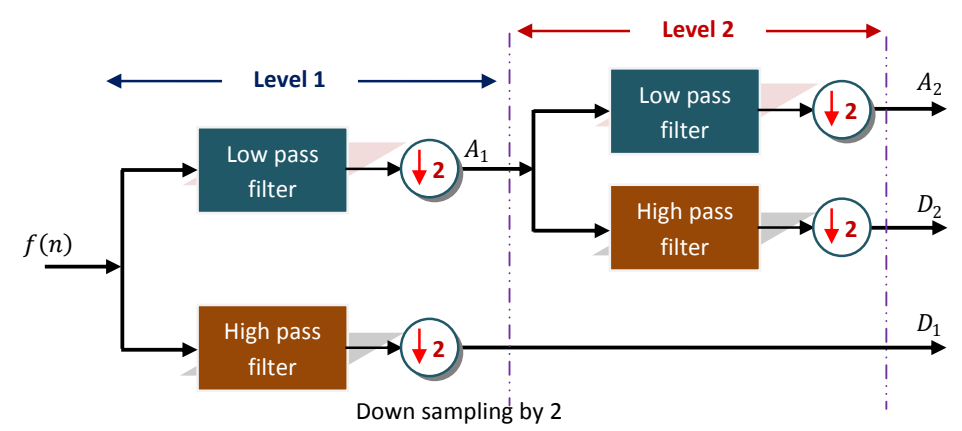

Fig. 1. Two-level DWT analysis.

\section{RMS Value Definition based on the Discrete Wavelet Transform}

The most common way to describe or analyze power quality events is by the measurement of the RMS value of voltage or current. According to [17], the RMS value of an electrical magnitude $f(t)$ of period $T$ (e.g., voltage) is determined by

$$
F=\sqrt{\frac{1}{T} \int_{t}^{t+T} f^{2}(t) d t}
$$

A particular case where the waveform the periodic signal $f(t)$ is sinusoidal it's RMS values is given by 


$$
F=\frac{F_{m}}{\sqrt{2}}
$$

where $F_{m}$ is the maximum amplitude of the periodic sinusoidal signal $f(t)$. However, real voltage and current signals are not ideally sinusoidal and will contain harmonics. In this case the voltage and current can both be expressed as a Fourier series as

$$
f(t)=\sum_{h} F_{h} \sin \left(2 h \pi \mathrm{f} t-\alpha_{h}\right)
$$

where $h$ is an existing harmonics order, $\alpha_{h}$ and $F_{h}$ are respectively the phase angle and the maximum amplitude of the $h^{t h}$ harmonics order and $\mathrm{f}=1 / T$ represents the frequency. The RMS values in Fourier domain is given by

$$
F=\sqrt{\sum_{h} \frac{F_{h}^{2}}{2}}
$$

The continue signal $f(t)$ is digitized to get $f(n)$, where $n=0,1,2, \cdots, 2^{N-1}$ for the period $T$. The RMS value of the digitized signal $f(n)$ is given as follows

$$
F=\sqrt{\frac{1}{2^{N}} \sum_{n=0}^{2^{N}-1} f^{2}(n)}
$$

In the Wavelet domain, the signal $f(t)$ is described by its Wavelet coefficients as

$$
f(t)=\sum_{k} c_{j_{0}, k} \times \phi_{j_{0}, k}(t)+\sum_{j \geq j_{0}} \sum_{k} d_{j, k} \times \psi_{j, k}(t)
$$

where $d_{j, k}$ and $c_{j_{0}, k}$ are the detail and approximation coefficients of the signal, respectively. $j$ is the frequency sub-band and $j_{0}$ is the lowest frequency sub-band (base-band). $\psi_{j, k}(t)$ is the Wavelet function (mother Wavelet) that the high-pass filter is derived form and measures the details. $\phi_{j_{0}, k}(t)$ represents the scaling function associated to the mother Wavelet that the low-pass filter is derived form and delivers a smoothed version of the input signal.

The RMS value of the digitized signal $f(n)$ in the Wavelet domain calculated using the Wavelet coefficientsis given as follows

$$
F=\sqrt{\left.\frac{1}{2^{N}} \sum_{k=0}^{2 j_{0}-1} c_{j_{0}, k}^{2}+\sum_{j \geq j_{0}}^{N-1} \sum_{k=0}^{j}-1 d_{j, k}^{2}\right)}
$$

\section{Numerical Simulation}

In order to evaluate the proposed approach accuracy, MATLAB has been used to compare the results of this method with analytical approach, which consists of the application of the definition given by the equation (8). Initially, digital current and voltage signals have been generated to be used as input signals in the proposed method of calculation. Secondly, both original waveforms are decomposed by using of discrete Wavelet transform method. Finally, the calculation of the RMS values using equation (10) have been proceeded. The proposed RMS calculation system based on DWT is illustrated in Fig 2. 


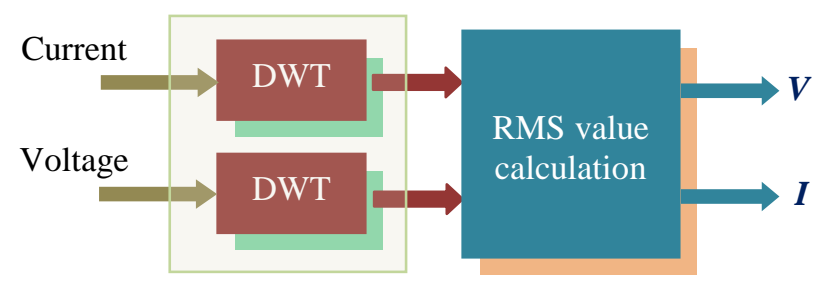

Fig. 2. Proposed structure for RMS calculation system based on DWT.

\section{A. The input signals}

In this simulation, two input signals have been used (current and voltage). The input signals are composed of the fundamental and the odd harmonics order up to 11, where each order has its own amplitude and phase.

In all cases, the voltage and current waveform has four cycles of $50 \mathrm{~Hz}$ with a sampling frequency of $25600 \mathrm{~Hz}$ or 512 points per cycle. This means that the original waveform has a length of 2048 points $(\mathrm{N}=11)$ during the observation period of $4 * T=4 / 50=0.08 \mathrm{sec}$.

According to Nyquist sampling theory, the maximum frequency that a sampled signal could faithfully be represented is equal to half its sampling frequency witch in our case is $12800 \mathrm{~Hz}$ [18-20]. The sampling frequency is largely sufficient where the frequency content of the input signal is up to the 11th harmonic which is $550 \mathrm{~Hz}$ on the 50 $\mathrm{Hz}$ line frequency.

The voltage signals that were chosen and used for the simulation is defined as

$$
\begin{aligned}
v(t)= & 311 \sin (100 \pi t)+20 \sin \left(300 \pi t+\frac{\pi}{2}\right)+ \\
& 12 \sin \left(500 \pi t+\frac{\pi}{3}\right)+5 \sin \left(700 \pi t+\frac{\pi}{8}\right)+ \\
& 3 \sin \left(900 \pi t+\frac{\pi}{6}\right)+0.5 \sin \left(1100 \pi t+\frac{\pi}{5}\right)
\end{aligned}
$$

As regards of the current, three cases are studied. For each case, different signal is generated. The three generated signals are identical in magnitude but different in phase (inductive, Capacitive and Resistive) and are given by the Eq. (12) for inductive current, Eq. (13) for capacitive current and Eq. (14) for resistive current.

$$
\begin{gathered}
i(t)=15 \sin \left(100 \pi t-\frac{\pi}{6}\right)+5 \sin \left(300 \pi t-\frac{\pi}{5}\right)+ \\
4 \sin \left(500 \pi t-\frac{\pi}{7}\right)+3 \sin \left(700 \pi t-\frac{\pi}{8}\right)+ \\
\sin \left(900 \pi t-\frac{\pi}{10}\right)+2 \sin \left(1100 \pi t-\frac{\pi}{12}\right) \\
c(t)=15 \sin \left(100 \pi t+\frac{\pi}{6}\right)+5 \sin \left(300 \pi t+\frac{\pi}{5}\right)+ \\
4 \sin \left(500 \pi t+\frac{\pi}{7}\right)+3 \sin \left(700 \pi t+\frac{\pi}{8}\right)+ \\
\sin \left(900 \pi t+\frac{\pi}{10}\right)+2 \sin \left(1100 \pi t+\frac{\pi}{12}\right) \\
r(t)=15 \sin (100 \pi t)+5 \sin (300 \pi t)+ \\
4 \sin (500 \pi t)+3 \sin (700 \pi t)+ \\
\sin (900 \pi t)+2 \sin (1100 \pi t)
\end{gathered}
$$

\section{B. The Wavelet decomposition of the input signals}

Sampled data is decomposed with the discrete Wavelet transform. A Daubechies mother Wavelet (db2) has been chosen in the present work, which means that the low and high pass filters of the decomposition have four coefficients. Although, currently there is no rule that helps choosing which mother Wavelet to use, it has been observed in recent 
study and research, that the Debauchees mother Wavelet is most stable, and the signal does not lose any information during the reconstruction process [21]. The maximum decomposition level that a signal can reach depends on the number of samples per period and the filters coefficients number [22]. In the simulation, the last decomposition level is set to four with sampling frequency of $25600 \mathrm{~Hz}$, thus decomposing the signal four-times would divide the band into [0 , $1600,3200,6400,12800] \mathrm{Hz}$.

The four harmonics distorted signals and its respective decomposition results are shown in figures 3, 4, 5 and 6, respectively. In all four figures the original signal is on top and its four levels decompositions are below.
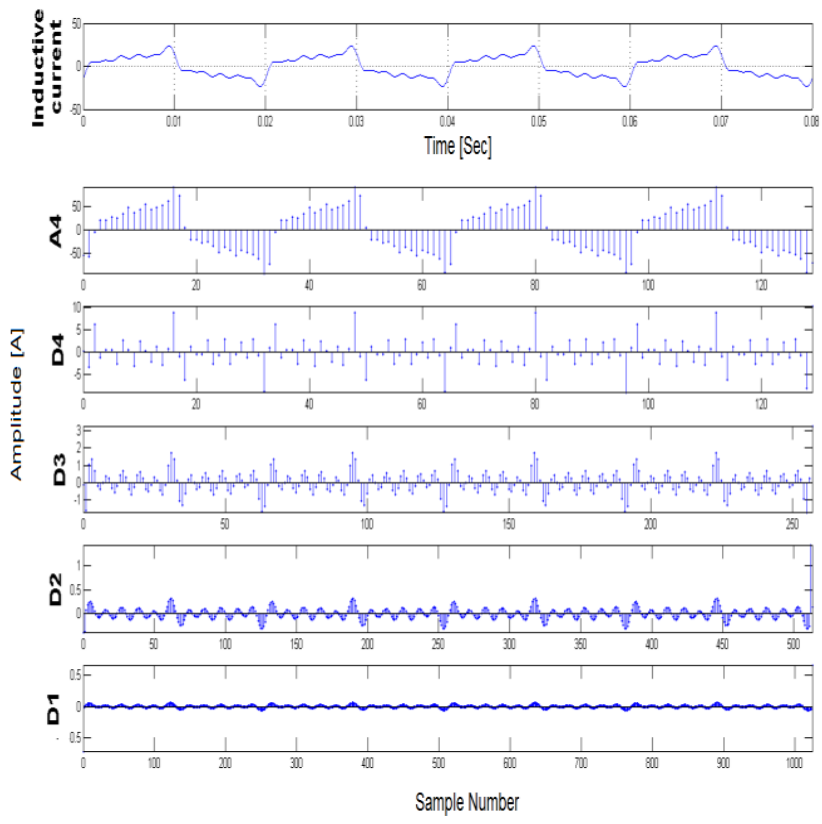

Fig.3. Wavelet decomposition of inductive current signal $i(t)$.
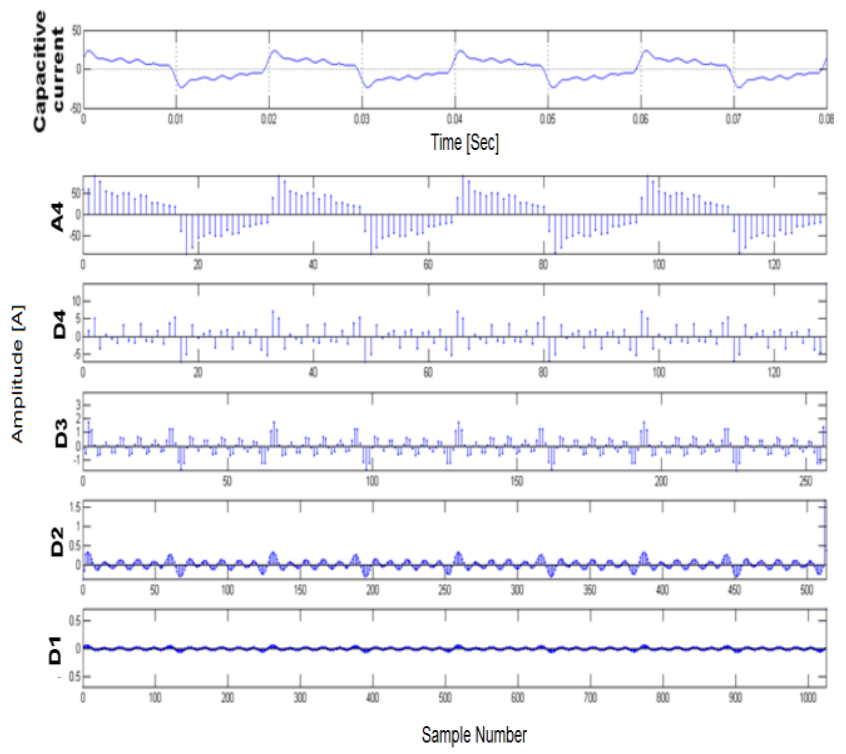

Fig.4. Wavelet decomposition of capacitive current signal $c(t)$.

All four input signals harmonic components do not exceed the 11 th order $(550 \mathrm{~Hz})$ which means that all harmonics are falling inside the band of $[0,1600] \mathrm{Hz}$. In other words, those frequencies are included in the approximation and in the fourth detail decomposition coefficients.

The rest of the bands ideally should not display any energy relating to these harmonics. However, it has been observed that a certain amount of harmonics energy leaks to higher-frequency bands D3, D2 and D1, this phenomenon is called filter leakage. The easiest way to reduce filter leakage is to use longer-length filters, but it yields to larger computation time. 

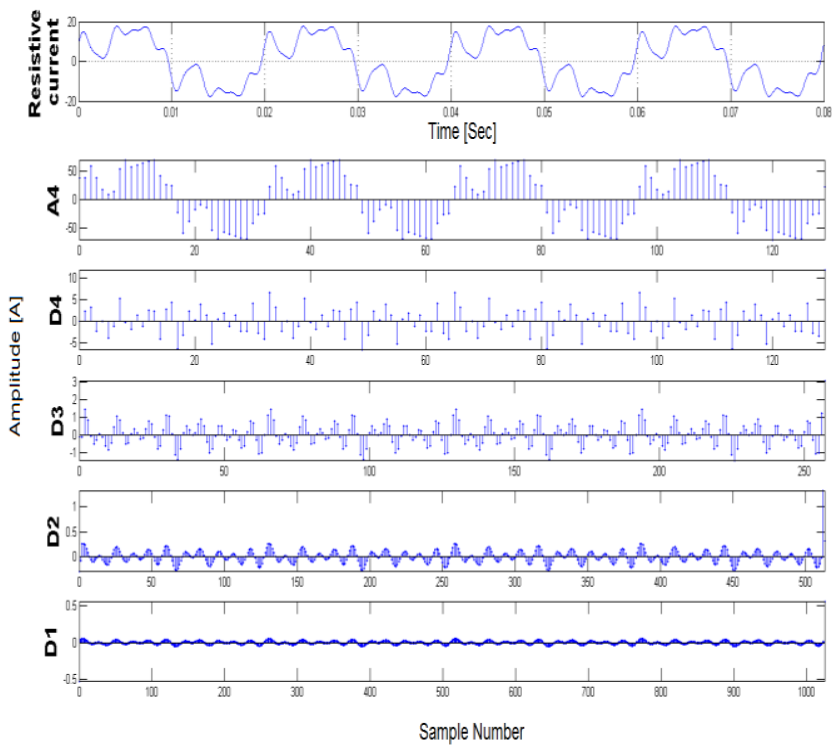

Fig. 5. Wavelet decomposition of resistive current signal $r(t)$.
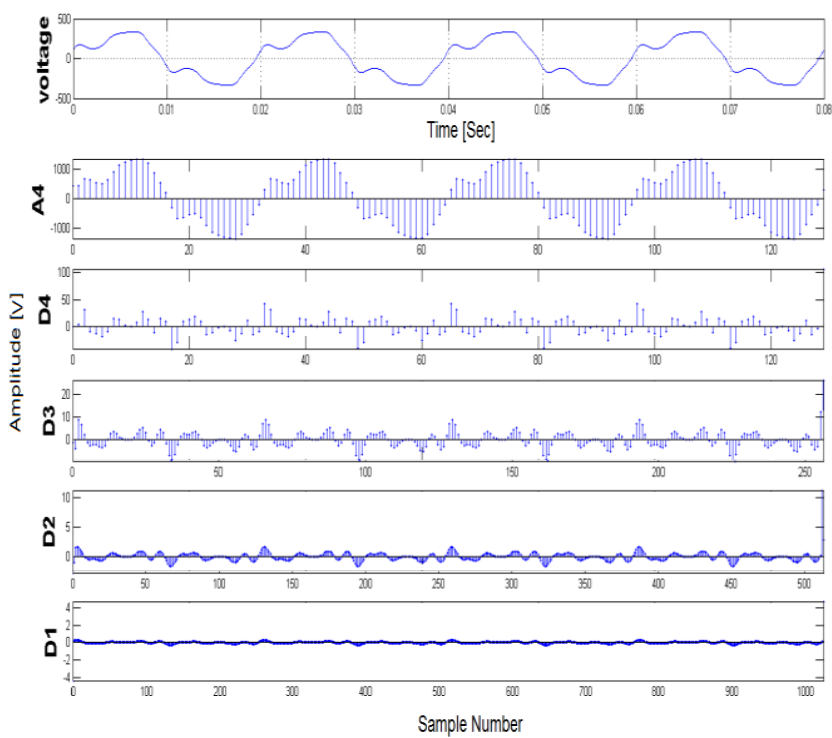

Fig. 6. Wavelet decomposition of voltage signal $v(t)$.

\section{Simulation results}

The proposed method for the calculation of the RMS values based on equation (10) is compared with the analytical approach which consists of the application of the definition given by equation (8).

Tables 1 illustrates the results for the analytical approach and the values calculated using DWT approach with the error according to the four signals, were V, I, C and R represent the RMS values of the voltage, the inductive current, the capacitive current and the resistive current signals, respectively.

Table 1. Results for the analytical and the DWT approaches.

\begin{tabular}{cccc}
\hline Inputs & Analytic results & DWT results & Error \% \\
\hline$V$ & $228.4207 \mathrm{~V}$ & $228.8562 \mathrm{~V}$ & 0.1907 \\
$I$ & $11.8322 \mathrm{~A}$ & $11.9552 \mathrm{~A}$ & 1.0403 \\
$C$ & $11.8322 \mathrm{~A}$ & $11.9780 \mathrm{~A}$ & 1.2324 \\
$R$ & $11.8322 \mathrm{~A}$ & $11.8931 \mathrm{~A}$ & 0.5147 \\
\hline
\end{tabular}

The simulation results as given by Table 1 shown that the error between the analytical results and the DWT results does not exceed $1.3 \%$ in all four cases which validate the theoretical presentation of the method of calculating described above. 
The error in the DWT approach results is due to the boundary or edge effect. Boundary effects are very common in the processing of finite-length signals. They are caused by incomplete data in the boundary regions of finite-length signals.

To reduce the edge effect the number of filter coefficients must be reduced and the number of sample per period must be increased. Truncating the unfavorable results at boundaries after convolution between signal and the Wavelet filters is another good solution to edge effect.

\section{Proteus Simulation}

The Proteus simulation set up of the proposed method includes several modules such as power supply, current and voltage sensors, microcontroller, Liquid Crystal Display (LCD) and load. These modules are integrated in a system block diagram as shown in Fig. 7.

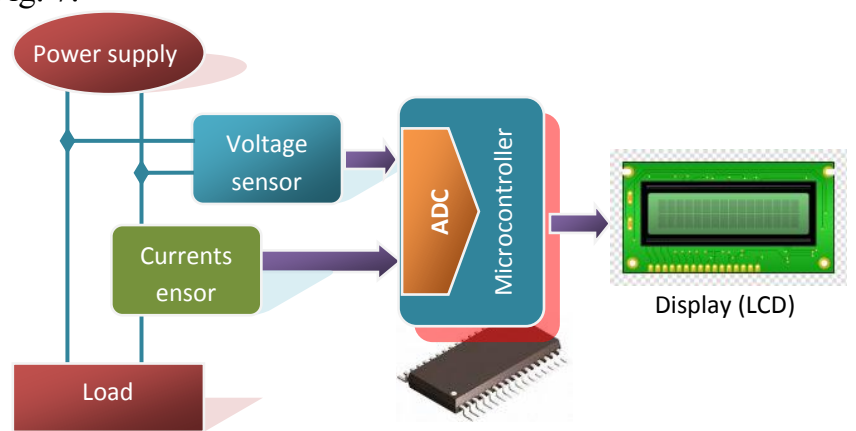

Fig. 7. Block diagram of the proposed RMS calculation system based on DWT.

\section{A. Power supply and load}

Three alternative generator connected in series have been used to produce a waveform that contains a fundamental and tow harmonics order the third and fifth. The equation representing the power supply is given by

$$
\begin{aligned}
v(t)= & 311 \sin (100 \pi t)+50 \sin (300 \pi t)+ \\
& 20 \sin (500 \pi t)
\end{aligned}
$$

As regard the load we used a $5 \mathrm{mH}$ inductance and a variable resistance to produce a phase shift between current and voltage waveform.

\section{B. Voltage sensor}

For the voltage measurement a voltage divider sensor has been used with a ratio of 200 to 1 . The microcontroller has an input voltage range of 0 to $5 \mathrm{~V}$ DC supply voltage (Vcc). Thus, sampled AC signal needs to be biased at Vcc/2. However, the DC bias voltage needs to be removed in order to calculate RMS value of the voltage and current [23]. The power supply voltage peak is $281 \mathrm{~V}$; thus the resistor divider output peak is $1.405 \mathrm{~V}$. To meet the ADC module requirements an Op-Amp IC has been included for buffering and level shifting the signal so the zero point will be centered at $2.5 \mathrm{~V}$. The equation that relates the input and output signals of the voltage sensor is given by

$$
V_{\text {out }}=\frac{\left(3 V_{\text {in }}\right)+1000}{400}
$$

where $V_{\text {in }}$ represents the input signal of the microcontroller and $V_{\text {out }}$ represents the voltage of the power supply.

\section{Current sensor}

For the current measurement, an ACS712 Hall Effect transducer has been used. This sensor design has various advantages such as DC currents measurement ability and its output is linear across the entire range of measured currents thanks to the closed loop design. In addition these types of sensors provide a galvanic isolation between the power supply and the electronics components. Fig. 8 schematizes the power supply and load in addition of voltage and current signals acquisition and conditioning are illustrated. The obtained current and voltage waveforms are displayed by fig. 9. 


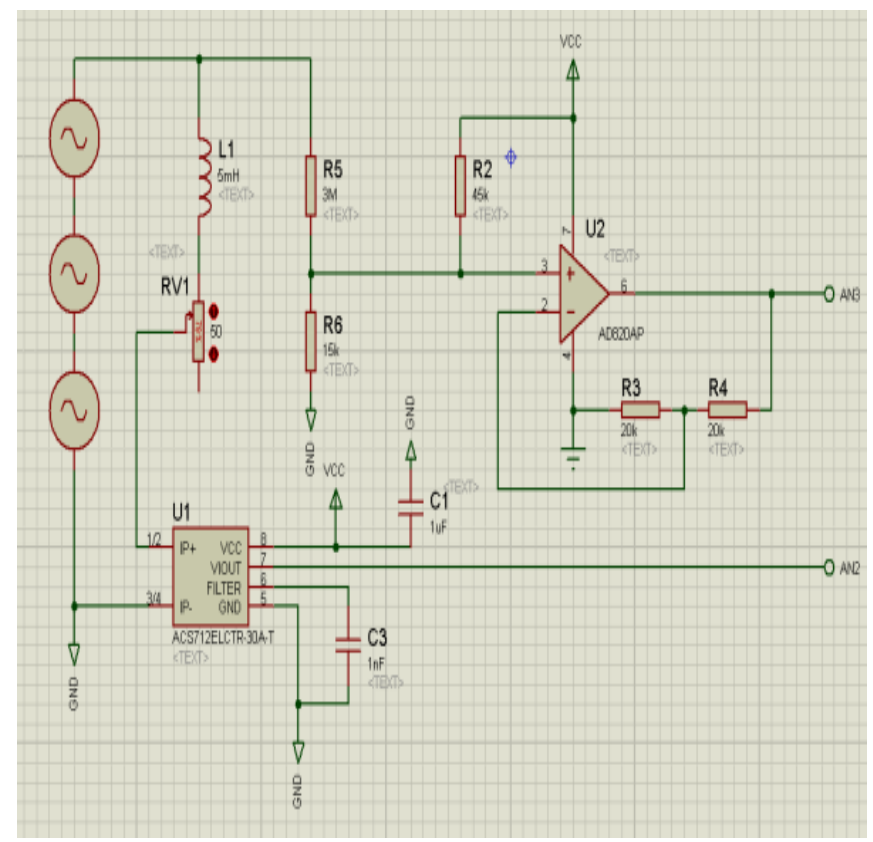

Fig. 8. Power supply and load in addition of voltage and current signals acquisition and conditioning.

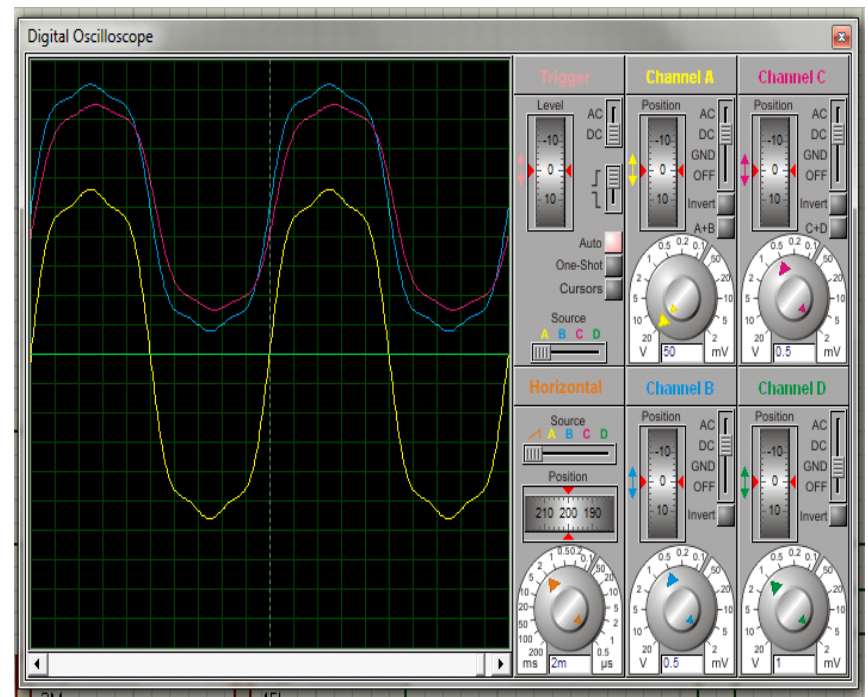

Fig. 9. Simulation of current and voltage waveforms. (Yellow $\rightarrow$ Voltage, Blue $\rightarrow$ Voltage image and Red $\rightarrow$ Current image)

\section{Microchip dsPIC33FJ32GP202 microcontroller}

In the proposed system (figure 10), a Microchip dsPIC33FJ32GP202 microcontroller has been adopted as calculator. It has the ability to operating at a clock frequency of $40 \mathrm{MHz}$, it possesses 16-bit modified Harvard architecture with the support for digital signal processing and includes a 256 Kbytes of program ROM and 30 Kbytes of SRAM [24].

The dsPIC33FJ32GP202 has one ADC module which supports up to 13 analog input channels with either 10-bit or 12-bit resolution. In addition the ADC module possess four sample and hold that offer the ability to make simultaneous samples, which is very important advantage for the precision of the measurements. The clock frequency of the dsPIC is $40 \mathrm{MHz}$ and 10 bit analogue-to-digital converter with simultaneous sampling is used to convert the test signal to discrete form. The ADC sampling rate is set at $400 \mathrm{~Hz}$ ( 8 samples/cycles) by use of timer 3 , which will trigger the end of sampling and starts the conversation of the desired analog inputs in this case AN2 and AN3. 


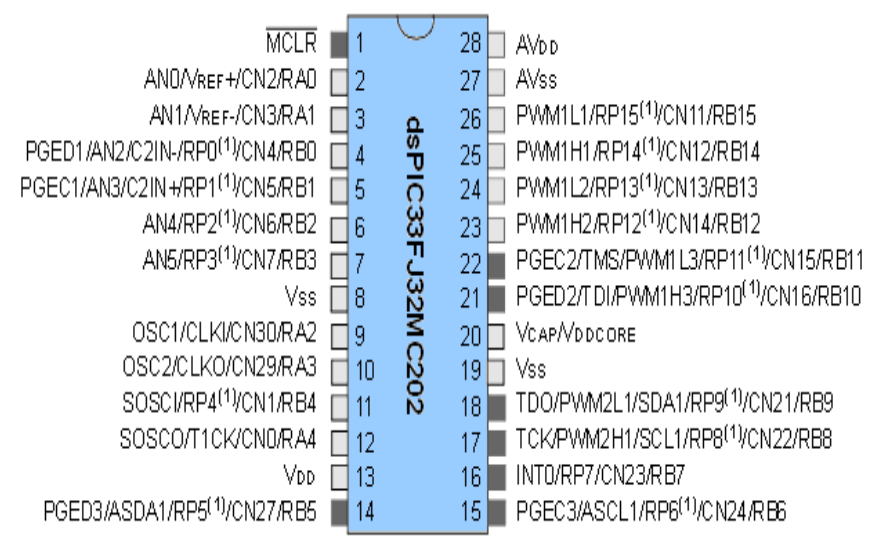

(a)

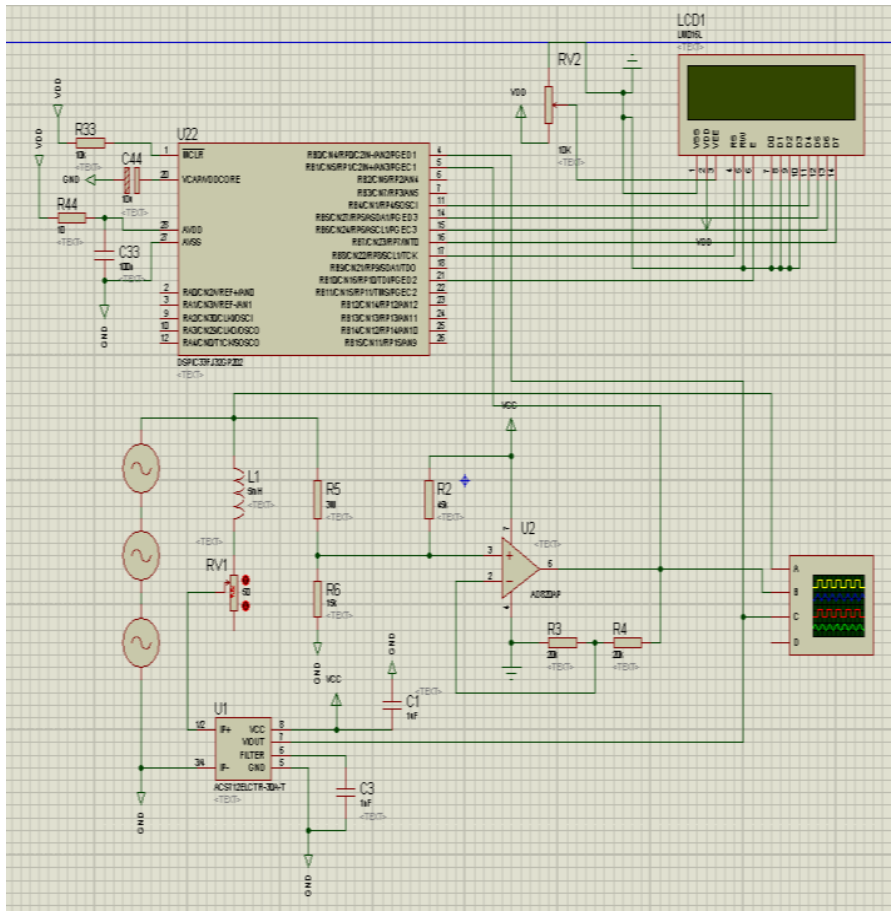

(b)

Fig. 10. Proteus simulation of the RMS calculation system based on dsPIC and DWT.

\section{E. Programming the dsPIC33FJ32GP202}

There are four main phases involved in this development of the RMS calculation system as shown in Figure 11, which are; Initialization process of devices used in this application, data acquisition, data process and data analysis stages. The last phase concerns the displaying of the obtained results. Each phase is further divided into several stages which include initializing, modelling, analysing and computing.

Programming the dsPIC is realized by using a $\mathrm{C}$ programming language tool to write and compile the code. The program starts with the initiation part where it first set the LCD module connection pins. Then, the program configures the ADC module using its three configuration registers AD1CON1, AD1CON2 and AD1CON3. To set the channels and the inputs that will be sampled, the program uses the ADC input select registers AD1CHS0 and AD1CHS123 in addition to the ports configuration register AD1PCFG.

Finally, the program initiates the Timer 3 module to set the conversation rate using the T3CON register. In the end of the initiation of the ADC and Timer 3 modules, the program disables there interruption and clear the interruption flag using IFS0 and IEC0 registers.

Once the initiation part is finished the program starts an infinite loop so the instruction within this loop will be repeated infinitely. The loop contains three set of instructions first the acquisition instructions were the program takes the converted sample from the ADC buffer AD1Buf and puts it in its appropriate array (current or voltage). 
Once the acquisition process is done, the program starts the calculation of the DWT coefficients and then uses those coefficients to calculate the RMS values. Finally the program converts the numerical values to texts and sends them to the LCD module so it can be displayed.

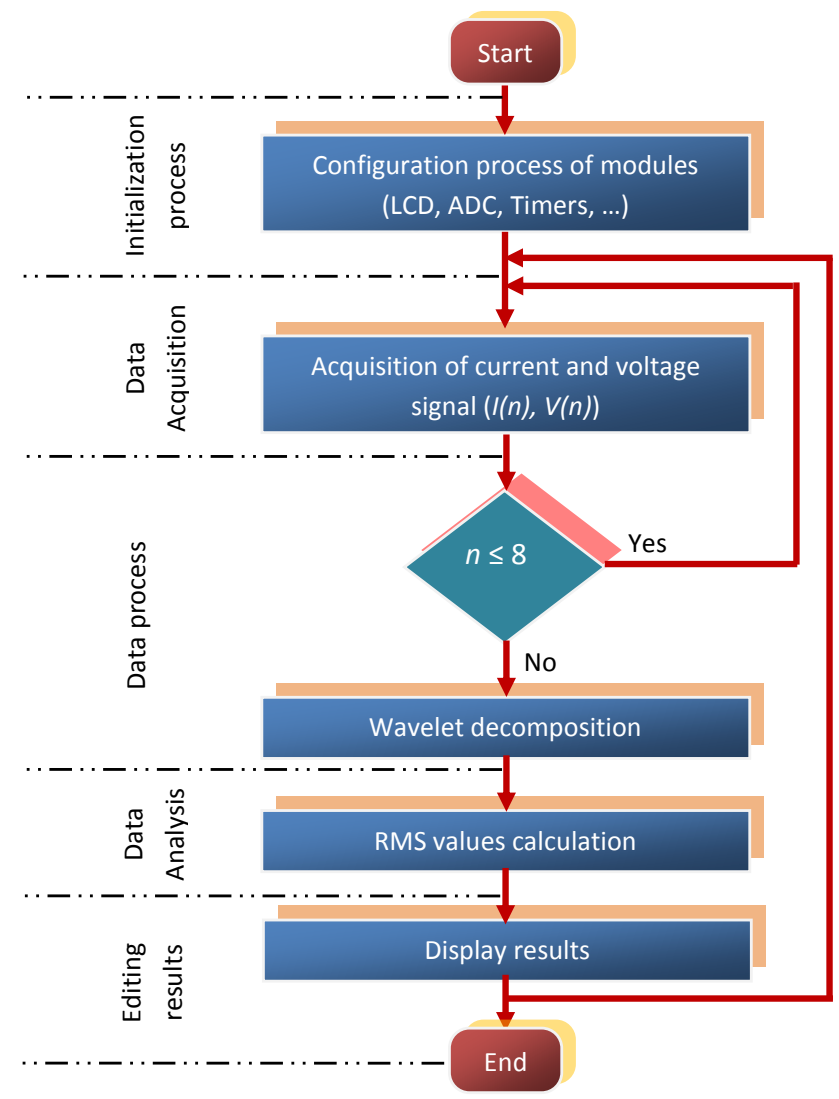

Fig. 11. Flowchart for RMS calculation based on dsPIC and DWT.

\section{F. Experimental results}

Once the algorithm was implemented, we run the system to obtain voltage and current calculated with the proposed method. The LCD displays those results, where the voltage is placed on the top line and in the bottom line the current. An ammeter and a voltmeter were installed to be used as reference to compare the results. The exact values of the current and voltage have been calculated analytically using the load and the power supply values.

The Analytic value has been used to calculate the relative error for both the proposed method and the meters. Figure 10 shows the configuration used in this simulation. The voltage of the power supply is constant but the current consumption of the load is varied through a variable resistance RV1.

Tables 1 and 2 show simulation results of the voltage and different values of current obtained from the proposed method, the Analytic results and the voltage and ammeter with their respective relative error.

Table 2. Comparison of Proteus simulation results for voltage.

\begin{tabular}{|c|c|c|c|c|c|}
\hline & \multirow{2}{*}{$\begin{array}{l}\text { Proposed } \\
\text { method } \\
\text { (V) }\end{array}$} & \multirow{2}{*}{$\begin{array}{l}\text { Analytic } \\
\text { results } \\
\text { (V) }\end{array}$} & \multirow{2}{*}{$\begin{array}{l}\text { Voltm- } \\
\text { eter } \\
\text { (V) }\end{array}$} & \multicolumn{2}{|c|}{ Relative error \% } \\
\hline & & & & Prop-met & Voltmeter \\
\hline $\begin{array}{c}\text { RMS } \\
\text { Voltage }\end{array}$ & 220.43 & 223.18 & 224 & 1.23 & 0.37 \\
\hline
\end{tabular}

Tables 2 and 3 illustrate the results for the proposed method,volt and ammeter and the true value calculated using the Analytic method and their respective relative error. The errors results errors are generally quite small where it does not exceed $1.23 \%$. This proves that the proposed RMS calculation method using the discrete Wavelet transform is correct. 
Table 3. Comparison of Proteus simulation results for current.

\begin{tabular}{ccccccc}
\cline { 5 - 6 } & \multicolumn{5}{c}{ Current RMS } \\
\hline $\begin{array}{c}\text { Resistor } \\
\text { value }\end{array}$ & Proposed & Analytic & Ammeter & \multicolumn{2}{c}{ Relative error \% } \\
\cline { 5 - 6 }$(\Omega)$ & method (A) & results (A) & $(\mathrm{A})$ & Prop-met & Ammeter \\
\hline 7.5 & 29.0341 & 29.0042 & 29.1 & 0.103089 & 0.330297 \\
10 & 21.9779 & 21.9869 & 22 & 0.040933 & 0.059581 \\
12.5 & 17.6618 & 17.691 & 17.7 & 0.165056 & 0.050873 \\
15 & 14.74 & 14.7769 & 14.8 & 0.249714 & 0.156325 \\
17.5 & 12.6481 & 12.6885 & 12.6 & 0.318399 & 0.697482 \\
20 & 11.0662 & 11.1155 & 11.1 & 0.443525 & 0.139445 \\
22.5 & 9.8232 & 9.8884 & 9.91 & 0.659358 & 0.218438 \\
25 & 8.8569 & 8.9048 & 8.89 & 0.537912 & 0.166202 \\
27.5 & 8.0437 & 8.0988 & 8.11 & 0.680348 & 0.138292 \\
30 & 7.3912 & 7.4263 & 7.42 & 0.472645 & 0.084834 \\
32.5 & 6.8022 & 6.8568 & 6.86 & 0.79629 & 0.046669 \\
35 & 6.3205 & 6.3684 & 6.36 & 0.752151 & 0.131901 \\
37.5 & 5.9073 & 5.9448 & 5.94 & 0.630803 & 0.080743 \\
\hline
\end{tabular}

Fig. 12 show the relative error calculated using the analytic result for the proposed method and the Ammeter. The error does not exceed $1 \%$ in all cases which is good but the method can be ameliorated by using better sensor, greeter $\mathrm{ADC}$ resolution and more simples per period.

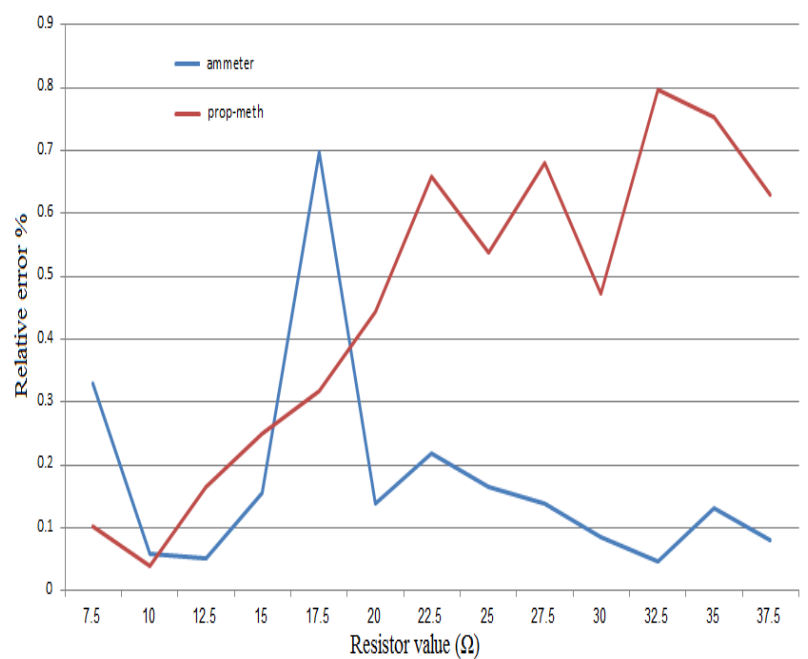

Fig. 12. Comparison between relative error of proposed method and Ammeter.

\section{Conclusion}

This paper focuses on the proposed RMS calculation system based on fast discrete Waveletand implemented by using a dsPIC-type microcontroller. The proposed method has the advantage of real-time calculation of distorted signals which can be implemented in energy meters. The system is simulated with MATLAB and implemented in a dsPIC using Proteus ISIS to validate the feasibility and effectiveness of the proposed method. The MATLAB simulation results have been compared to the analytic results and have shown a good precision where the error does not exceed $2 \%$ in all simulated cases. The calculation method is successfully implemented on Proteus ISIS and the results have been compared to a traditional volt and ammeter. The implementation results are good and can be improved by increasing the number of samples per period, the ADC output resolution and the DWT filters coefficients number.

\section{References}

[1] S. Jaiswal, M.S. Ballal, "FDST-based PQ event detection and energy metering implementation on FPGA-in-the-loop and NILabVIEW,' The Institution of Engineering and Technology Science, Measurement \& Technology, vol. 11, no. 4, pp. 453-463, 2017.

[2] L. Angrisani, P. Daponte, M. D'apuzzo, A. Testa, “'A Measurement Method based on the Wavelet Transform for power quality analysis,’ IEEE Trans. On Power Delivery, vol. 13, pp. 990-998, 1996. 
[3] W.-K Yoon, M. J. Devaney, “Power Measurement using the Wavelet Transform,''IEEE Trans. On Instrumentation and Measurement, vol. 47, no.5, pp. 1205- 1210, October 1998.

[4] W.-K Yoon, M. J. Devaney, “Reactive Power Measurement using the Wavelet Transform,', IEEE Trans. On Instrumentation and Measurement, vol. 49, no.2, pp. 1205-1210, April 2000.

[5] D. Borras, M. Castilla, N. Moreno, J.C. Montano, “Wavelet and Neural Structure: A New Tool for Diagnostics of Power System Disturbances,’ IEEE Trans. On Industry Applications, vol. 37, no. 1, pp. 184-190, Jan./Febr. 2001.

[6] E.Y. Hamid, R. Mardiana, Z.I. Kawasaki, "Method for RMS and power measurements based on the wavelet packet transform," IEE Proceedings - Science, Measurement and Technology, vol. 149, no. 2, pp. 60-66, 2002.

[7] F.Vatansever, A.Ozdemir, "A new approach for measuring RMS value and phase angle of fundamental harmonic based on Wavelet Packet Transform," Electric Power Systems Research, vol. 78, no. 1, pp. 74-79, 2009.

[8] J. M.Flores-Arias et al. "A memory-efficient true-RMS estimator in a limited-resources hardware,' Energies, vol. 12, pp. 2-18, 2019.

[9] S.P. Kambleet al."A Comparative Analysis for Selection of Appropriate Mother Wavelet for Detection of Stationary Disturbances," Journal of The Institution of Engineers (India): Series B, vol. 98, pp. 533-540, 2017.

[10] J. Modarresi, R.A. Hooshmand, S.M Nosratabadi, "Enhancement of power measurement using a modified method based on wavelet with preprocessing: electric arc furnace case study," Transactions of the Institute of Measurement and Control, vol 37, no. 9, pp. 1095-1108, 2015.

[11] M.O. Oliveira, J.H. Reversat and L.A Reynoso, "Wavelet Transform Analysis to Applications in Electric Power Systems,' in "Wavelet Transform and Complexity", IntechOpen Publisher, 2019. DOI: 10.5772/intechopen.85274

[12] C. S. Burrus, R. A. Gopinath, and H. Guo, "Introduction to Wavelets and Wavelet Transform: A Primer," Englewood Cliffs, NJ: Prentice-Hall, 1998.

[13] A.N. Akansu, R. A. Haddad,“Multi-resolution Signal Decomposition Transforms, Sub-bands, and Wavelets,” Second Edition, New Jersey Institute of Technology Newark, NJ, ACADEMIC PRESS, 2001.

[14] I. Daubechies, Ten Lectures on Wavelets, Philadelphia, PA, USA:Society for Industrial and Applied Mathematics, 1992.

[15] D. T. L. Lee, A. Yamamoto, "Wavelet analysis: theory and applications," Hewlett-Packard Journal, pp. 44-54, December 1994.

[16] S. Mallat, "A theory for multi-resolution signal decomposition: the Wavelet representation," IEEE Trans. on Pattern Analysis And Machine Intelligence, vol. 11, no. 7, pp. 674-693, 1989.

[17] International Electrotechnical Commission. IEC 61000-4-30: 2015. Electromagnetic compatibility (EMC)—Part 4-30: Testing and Measurement Techniques-Power Quality Measurement Methods; International Electrotechnical Commission: Geneva, Switzerland, 2015.

[18] Shannon, E. Claude, "Communication in the presence of noise,"Proceedings of the Institute of Radio Engineers. (January 1949). Reprint as classic paper in: Proc. IEEE, Vol. 86, No. 2, Feb 1998.

[19] H. Luke, "The origins of the sampling theorem," IEEE Comm. Mag., vol. 37, no. 4, pp. 106-108, Apr 1999.

[20] M. Mishali, Y.C. Eldar, "Blind Multiband Signal Reconstruction: Compressed Sensing for Analog Signals," Signal Processing IEEE Transactions on, vol. 57, no. 3, pp. 993-1009, March 2009.

[21] K. M. Siddiqui, K. Sahay, V. K. Giri, "Early; diagnosis of stator inter-turn fault in inverter driven induction motor by Wavelet transform," 2016 IEEE 1st International Conference on Power Electronics Intelligent Control and Energy Systems (ICPEICES), pp. 1-6, July 2016.

[22] C.S. Wang, "Power Disturbance Analysis via Discrete Wavelet Transform," Wseas Transactions on Power Systems, vol. 9, pp. 208-218, 2014

[23] L. Labib et al. "Design and implementation of low-cost universal smart energy meter with demand side load management," The Institution of Engineering and Technology Generation, Transmission \& Distribution, vol. 11 no. 16, pp. 3938-3945, 2017.

[24] Microchip, "High-Performance, 16-bit Digital Signal Controllers", DS70290F datasheet, 2003.

\section{Authors' Profiles}

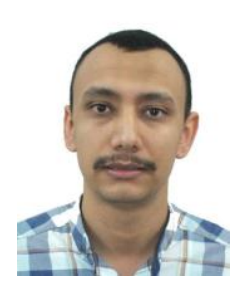

Hossam Eddine Guia is a Professor of Electrical Engineering at the Faculty of Sciences and Technology, Eloued University, Algeria. He received his Engineering degree in Electromechanics and the Magister degree in Mechatronics (2012) from Automation Department, Boumerdes University, Algeria. He is currently pursuing Doctorate $(\mathrm{PhD})$ degree at the Faculty of Sciences and Technology, Eloued University, Algeria. His current research interests include Renewable Energies, mechatronics, artificial intelligence, and programmable logic device.

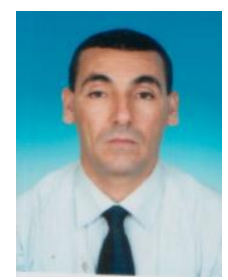

Dr. Ammar Soukkou is a Professor of Electronics at the Faculty of Sciences and Technology, Jijel University, Algeria. He received his Engineering degree, the Magister degree, and the Doctorate $(\mathrm{PhD})$ in Engineering Control (2008) from Electronics Department, Setif University, Algeria. He is, also, received the Habilitation à Diriger les Recherches (HDR) degree from Department of Electronics, Jijel University, Algeria. Since 2000 to 2005, he held different positions involved in industrial field and education at Skikda University, Algeria. Since 2005, he has been an Assistant Professor at the Electronics department, University of Jijel, Algeria. He is the author of various publications in international journals $\&$ proceedings. 
His current rechearch interests includes intelligent and advanced approcahes in modeling and control of Biotechnological and renewable energy processes, Fractional-order field, advanced optimization techniques, fractional-order chaotic systems and stability/robustness analysis of dynamical systems.

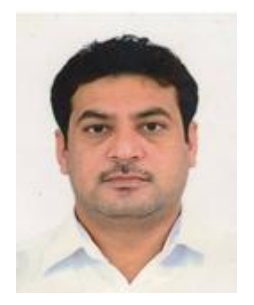

Dr. Redha Meneceur is a Professor of mechanical Engineering at the Faculty of Sciences and Technology, Eloued University, Algeria. He received his Engineering degree, the Magister degree, and the Doctorate $(\mathrm{PhD})$ in Industrial maintenance (2018) from mechanical Engineering Department, Skikda University, Algeria.

His current research interests includes Renewable Energies, mechatronics, Heat Source's Cooling, artificial intelligence.



Abdelkrim Mohrem is currently pursuing Doctorate $(\mathrm{PhD})$ degree at University of Boumerdes at the Faculty of hydrocarbons and chemistry, Algeria. He received his Engineering degree in Electromechanics and the Magister degree in Mechatronics (2012) from Automation Department, Boumerdes University, Algeria.

His current research interests includes Diagnostic, Smart Energy meter, Renewable Energies, mechatronics, artificial intelligence, programmable logic devices.

How to cite this paper: Hossam Eddine Guia, Ammar Soukkou, Redha Meneceur, Abdelkrim Mohrem, " Design and Implementation of Real Time RMS Measurement System based on Wavelet Transform Using adsPIC-type Microcontroller", International Journal of Image, Graphics and Signal Processing(IJIGSP), Vol.12, No.6, pp. 43-56, 2020.DOI: $10.5815 /$ ijigsp.2020.06.05 